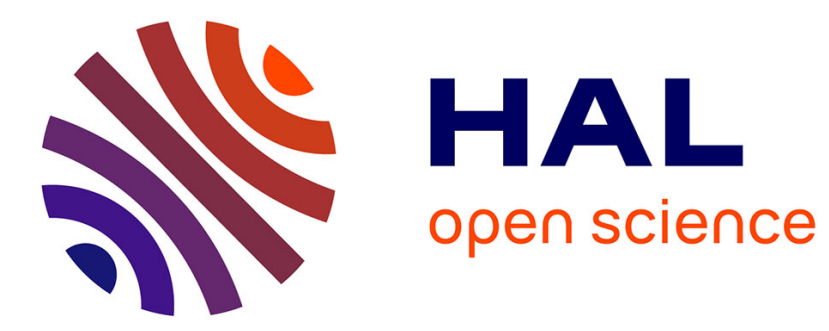

\title{
L'enseignement des langues vivantes à l'école élémentaire: éléments d'évaluation des effets au collège
}

\author{
Sophie Genelot
}

\section{To cite this version:}

Sophie Genelot. L'enseignement des langues vivantes à l'école élémentaire : éléments d'évaluation des effets au collège. 1996, pp.5. hal-03206871

\section{HAL Id: hal-03206871 \\ https://hal.science/hal-03206871}

Submitted on 28 May 2021

HAL is a multi-disciplinary open access archive for the deposit and dissemination of scientific research documents, whether they are published or not. The documents may come from teaching and research institutions in France or abroad, or from public or private research centers.
L'archive ouverte pluridisciplinaire HAL, est destinée au dépôt et à la diffusion de documents scientifiques de niveau recherche, publiés ou non, émanant des établissements d'enseignement et de recherche français ou étrangers, des laboratoires publics ou privés. 


\title{
les Notes de l'irédu
}

institut de recherche sur l'économie de l'éducation

\section{L'enseignement des langues vivantes à l'école élémentaire : éléments d'évaluation des effets au collège}

\author{
Sophie Genelot
}

En 1989, le ministère de l'Education Nationale a mis en oeuvre, à grande échelle, un plan d'expérimentation contrôlée d'enseignement des langues vivantes à l'école primaire : $10 \%$ des élèves de $\mathrm{CM}$ devaient être concernés dès la première année, plus de $30 \%$ en ont bénéficié au cours de la troisième année. Cette décision a amorcé, de fait, l'émergence d'une nouvelle discipline de l'école élémentaire, même si, à l'époque, la généralisation d'un tel enseignement n'était pas explicitement envisagée. Depuis la fin de la période expérimentale, en septembre 1992, son extension mesurée mais régulière, dans les écoles élémentaires, traduit une volonté politique affirmée.

L'expérimentation de 1989 s'appuyait, même si cela reste assez implicite dans les textes officiels, sur un postulat principal selon lequel plus on commence tôt l'apprentissage d'une discipline et plus celui-ci est long, meilleurs sont au final les résultats des élèves. Par ailleurs, elle visait de façon explicite une préparation des élèves à l'apprentissage des langues au collège. L'évaluation, dont il est question ici, a principalement porté sur cet objet : on a cherché à mesurer si une telle réforme avait contribué à l'amélioration effective de l'efficacité de l'enseignement des langues étrangères au niveau du second degré. Le second objectif de cette recherche a consisté, de façon complémentaire, à identifier et à décrire l'effet d'un certain nombre de facteurs pouvant déterminer, pour partie, l'efficacité de l'enseignement de l'anglais à l'école élémentaire et au collège.

L'échantillon (Académie de Dijon) compte 1000 élèves initiés à l'anglais à l'école élémentaire et 500 élèves témoins issus de 13 collèges. Il a servi de base à une enquête longitudinale qui a permis de suivre ces élèves, de septembre 1991 à juin 1993, au cours de leurs deux premières années de collège. La méthodologie employée s'appuie sur l'examen, à la fin de l'école primaire, des résultats en anglais des élèves qui ont bénéficié de l'expérimentation, ainsi que sur l'analyse de leurs résultats, comparés à ceux des élèves témoins, à des épreuves communes à l'issue des classes de $6^{\text {ème }}$ et de $5^{\text {ème }}$.
L'utilisation de la modélisation multivariée a permis d'estimer le poids respectif de différents groupes de variables dans l'explication des scores (dont la distribution a été standardisée à une moyenne de 100 et un écart-type de 15 ) et d'identifier quels sont les facteurs les plus déterminants du niveau atteint par les élèves.

\section{Les déterminants des acquisitions des élèves en anglais au primaire et au collège}

L'analyse des résultats conduit à identifier 3 familles d'éléments qui pèsent sur la construction des acquisitions des élèves en anglais. Les plus déterminants d'entre eux concernent les élèves eux-mêmes : les caractéristiques socio-démographiques des élèves de l'échantillon et leur "valeur scolaire" (appréhendée par l'intermédiaire de leur âge et de leur niveau de français à l'entrée en $6^{\text {ème }}$ ) contribuent, par exemple, à expliquer près de $27 \%$ de la variation des scores en fin de primaire. Le troisième groupe d'éléments est constitué par l'ensemble des conditions relatives à l'organisation matérielle et pédagogique de cet enseignement aux niveaux concernés (l'école élémentaire et la $6^{\text {ème}}$ ) : ces facteurs s'avèrent être responsables de 14,5\% des différences présentes en anglais entre les élèves à la fin de l'école primaire.

On peut distinguer deux types de résultats. Tout d'abord ceux qui font écho à des travaux de recherche utilisant la même démarche méthodologique et qui viennent confirmer, pour la plupart, certains effets observés à propos d'autres acquisitions scolaires à l'école primaire et au collège (Duru M. \& Mingat A., 1988).

Ainsi, il apparaît qu'à l'école primaire comme au collège les acquis des élèves en anglais se trouvent être soumis à des différenciations sociales de même nature que celles relevées à propos d'autres types d'acquisitions. Les élèves qui appartiennent aux classes socialement favorisées 
progressent davantage, toutes choses étant égales, au cours du cycle d'observation que les autres élèves ; ceux qui présentent déjà un "retard scolaire" à l'entrée en 6 ème, moins que les élèves "à l'heure". Par ailleurs, les résultats obtenus permettent, une nouvelle fois, de mettre en doute l'existence d'un effet de la taille de la classe sur les acquisitions des élèves puisqu'aussi bien en $6^{\text {ème }}$ qu'à l'école primaire, où la variété est importante dans l'échantillon (de 2 à 29 élèves), il n'apparaît pas de relation significative entre cette variable et le niveau atteint par les élèves.

Enfin, comme d'autres études empiriques l'ont également observé, le temps d'apprentissage s'avère être un facteur central dans l'explication des différences d'acquisitions observées en anglais entre les élèves à la fin de l'école primaire. Les analyses montrent que plus l'initiation à l'anglais a été longue, meilleur est le niveau obtenu par les élèves dans cette discipline : dix heures d'enseignement sont associées à un gain de 2,5 pts en moyenne. On observe, cependant, un seuil de saturation audelà duquel l'effet d'une heure d'enseignement supplémentaire ne produit plus qu'un impact modeste sur le niveau atteint par les élèves.

\section{Graphique $n^{\circ} 1$ \\ Relation entre durée totale d'initiation et score obtenu par les élèves en anglais à la fin de l'école primaire}

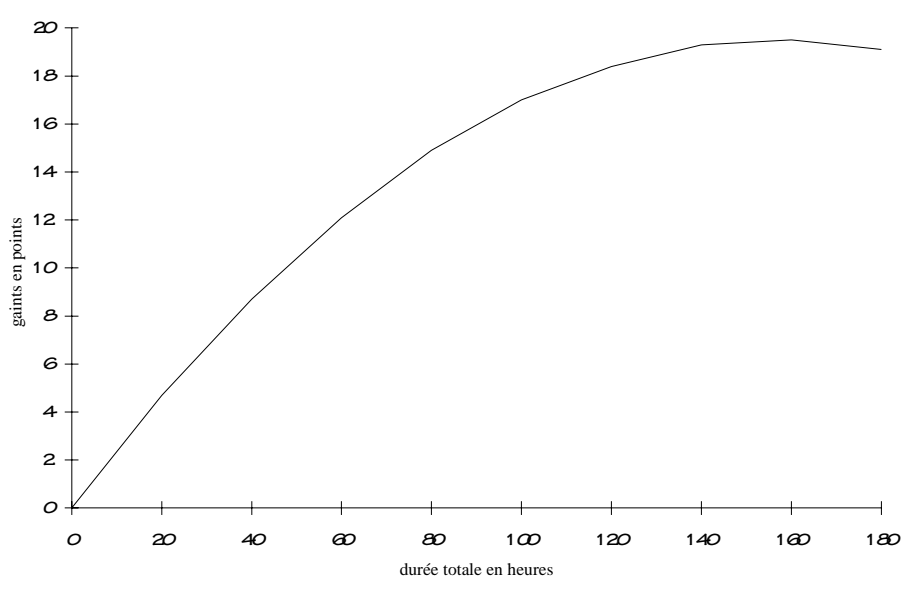

Dans cette recherche, le temps d'apprentissage a pu également être appréhendé sous l'angle de sa répartition hebdomadaire : la durée des séances a été introduite dans l'analyse (de 24 à 90 minutes sur l'ensemble des classes avec une moyenne à $51 \mathrm{mn}$ ). Il s'avère qu'à durée hebdomadaire donnée, plus les séances sont courtes, meilleures sont les acquisitions des élèves.
Notons également que des analyses complémentaires ont montré que l'impact de la durée d'apprentissage est plus important pour les élèves forts que pour les élèves faibles : il n'est donc pas certain qu'il suffirait, comme on l'entend communément parfois, de proposer une augmentation d'horaire aux élèves les plus en difficulté pour pallier à celles-ci, puisqu'il semble que ces élèves réussissent moins que les autres à "rentabiliser" la durée d'enseignement qui leur est offerte.

Le second type de résultats obtenus par cette recherche est relatif à des questions spécifiques à l'enseignement des langues étrangères ; ils constituent, pour certains, des contreparties empiriques aux débats à dominante plutôt idéologique qui existent en didactique des langues étrangères. Cette recherche permet d'identifier, par exemple, des liaisons non négligeables entre les différentes acquisitions dans le domaine des langues. Ceci s'observe d'une part, par la dépendance existant entre le niveau atteint par les élèves en anglais et leur niveau de maîtrise en français (meilleurs ils sont en français, meilleurs ils sont en anglais) mais également par la meilleure progression en anglais des élèves bilingues sur leurs camarades monolingues $^{1}$ (cet effet s'amenuise cependant avec le temps : il est plus important en primaire qu'il ne l'est en $6^{\text {ème }}$ et en $\left.5^{\text {ème }}\right)$.

La question de l'efficacité des différentes méthodes d'enseignement a été abordée selon la technique dite de la "boîte noire" qui considère l'emploi d'une méthode dans sa globalité et ne permet donc pas d'identifier les raisons pour lesquelles une méthode apparaît plus efficace qu'une autre.

En primaire, sur l'ensemble de l'échantillon, la moitié des enseignants suit régulièrement une méthode précise (12 méthodes ont été répertoriées). La pratique des autres peut être rapprochée : - soit d'une action de "sensibilisation à la langue" (28\%) ; - soit d'un enseignement à dominante "linguistique" et structuré recourant à plusieurs méthodes à la fois $(21 \%)$.

L'ensemble de ces méthodes peut être scindé en 3 groupes selon leurs effets respectifs sur les acquisitions des élèves : a) enseignement de type "linguistique" et utilisation de Muzzy in Gondoland ; b) ceux qui utilisent Stepping

\footnotetext{
${ }^{1}$ L'expression "élève bilingue" désigne ici des élèves étrangers ou d'origine étrangère (majoritairement d'Afrique du Nord) qui pratiquent régulièrement à la maison leur langue maternelle d'origine, ils sont $11,2 \%$ dans l'échantillon.
} 
stones ; c) les autres ${ }^{2}$. Comparés à ces derniers, les élèves du premier groupe gagnent 4 pts, ceux du second en perdent 4,1 . Des analyses complémentaires montrent également que certaines de ces méthodes d'enseignement sont inégalement efficaces en fonction de la durée d'initiation et du niveau scolaire (en français) des élèves auxquels elles s'adressent.

En $6^{\text {ème }}$, les méthodes d'enseignement (au nombre de quatre) présentent également une efficacité différente sur la progression des élèves en anglais au cours de l'année : Apple pie apparaît plus efficace que Channel $6(+4,8$ pts) tout comme Come in! (+ 3,8 pts). De plus, ces différentes méthodes ne sont pas toutes également efficaces selon qu'elles sont utilisées auprès d'élèves déjà initiés en primaire, ou d'élèves débutant l'apprentissage de l'anglais au collège. Il reste, cependant, que la contribution spécifique des méthodes d'enseignement à l'explication de la variance des scores est modeste (1,5\%, par exemple, en primaire).

La présente recherche apporte également quelques résultats à propos de l'efficacité de certaines pratiques pédagogiques déclarées. L'apport le plus significatif et le plus intéressant, car il permet de contribuer à un débat souvent très dogmatique, porte sur le positionnement des enseignants à propos de l'emploi de la langue française dans leurs cours. Il apparaît que ce n'est sans doute pas tant l'utilisation de la langue maternelle, en elle-même, qu'il est important de considérer, mais davantage l'usage qui en est fait, puisque ses différents modes d'emploi (pour enseigner la grammaire, donner des explications, gérer la vie quotidienne de la classe) produisent des effets différents sur les acquisitions des élèves.

Enfin, les résultats de l'analyse en fin de primaire montrent une efficacité particulière des instituteurs sur les acquisitions des élèves par rapport aux autres intervenants. Il convient, cependant, de mettre en garde contre toute interprétation insuffisamment distanciée de ce résultat. Il faut noter, en effet, que la recherche montre également que le niveau de formation des intervenants en anglais a un effet positif (non significatif) sur les acquisitions des élèves, même si cette caractéristique s'avère être moins pertinente, que le statut des intervenants, pour expliquer les différences de niveau. Ainsi, on peut penser que l'initiation conduite à l'école élémentaire, pour être pleinement efficace, nécessite, de la part des intervenants qui en sont responsables, en plus d'une maîtrise suffisante

\footnotetext{
${ }^{2}$ Ce groupe comprend les pratiques de type "sensibilisation", l'enseignant qui emploie Pick it up, et ceux qui emploient toutes les autres méthodes peu représentées dans l'échantillon des classes.
}

de la discipline enseignée, une bonne connaissance du public auquel ils s'adressent, puisque ce qui distingue principalement les instituteurs des autres catégories d'intervenants c'est que ce sont les seuls qui, dans cette situation, travaillent avec leur public habituel. Ce résultat est intéressant dans la mesure où la question du choix des intervenants pour conduire $1^{\prime} E L^{3}{ }^{3}$ est une décision institutionnelle délicate en raison de ses incidences pédagogiques et budgétaires.

\section{Impact de l'EPLV sur les acquisitions en anglais au collège}

Les différentes analyses comparatives du niveau atteint, en anglais, par les élèves initiés et par les élèves "témoins", montrent tout d'abord que l'expérimentation, telle qu'elle a été mise en oeuvre dans les classes de l'échantillon et telle qu'elle a été évaluée ici, laisse des traces pour le moins minimes dans les acquisitions des élèves initiés, mesurées à la fin des classes de $6^{\text {ème }}$ et de $5^{\text {ème }}$. La variable qui indique si un élève a ou non bénéficié de l'EPLV participe, en effet, de façon extrêmement marginale à expliquer les différences de progression des élèves au cours des deux premières années de collège $\left(2 \%\right.$ en $6^{\text {ème }}$, seulement $0,2 \%$ en $5^{\text {ème }}$ ).

Une première approche de son impact global révèle un effet légèrement positif en $6^{\text {ìme }}$ : les élèves initiés en primaire obtiennent, en moyenne et toutes choses égales par ailleurs, 1,6 pt de plus que les élèves "témoins". A la fin de la classe de $5^{\text {ème }}$, cependant, l'initiation ne présente plus aucun impact significatif sur la progression des élèves. Les résultats d'analyses complémentaires montrent que ce bénéfice moyen de l'initiation, observé en fin de $6^{\text {ème }}$, masque en fait des différences selon le niveau scolaire (niveau en français) des élèves : ce sont principalement les élèves les plus forts qui ont la capacité de mobiliser, au cours de l'année de $6^{\text {ème }}$, les connaissances acquises en anglais au cours de l'école primaire ; pour les élèves les plus faibles, le fait d'avoir bénéficié de l'EPLV n'a aucune incidence significative sur leurs résultats au collège.

Enfin, la présente recherche a permis de mettre en évidence quelques effets "pervers" dûs à cette expérimentation. Le premier d'entre eux est contextuel. Il est la conséquence du fait que l'initiation n'est pas

\footnotetext{
${ }^{3}$ EPLV : Enseignement Pré-scolaire des Langues Vivantes, terme emprunté à O'Neill C. (1993).
} 
généralisée : on constate, en effet, que les élèves débutant l'apprentissage de l'anglais en $6^{\text {ème }}$ en étant mêlés à des élèves initiés obtiennent, de ce fait, un niveau d'anglais inférieur (de 4 pts) à celui qu'ils auraient pu atteindre s'ils avaient été scolarisés avec d'autres débutants ${ }^{4}$. On constate également que les élèves, initiés à l'anglais en primaire, qui ont été regroupés entre eux en $6^{\text {̀̀me }}$ ont davantage progressé que ceux d'entre eux qui ont été mêlés à des débutants.

L'autre conséquence de l'expérimentation est beaucoup plus fondamentale, nous semble-t-il, et renvoie aux questions d'arbitrage entre les différentes disciplines de l'école primaire. La recherche a montré, en effet, que l'introduction de l'enseignement de l'anglais à l'école primaire est significativement liée à une moindre réussite des élèves en français à la fin de ce cursus. Cet effet ne semble pas pouvoir être imputé seulement à la présence de l'expérimentation mais bien à la durée de l'initiation (graphique $\mathrm{n}^{\circ} 2$ ), ce qui permet de soupçonner que c'est, sans doute, par l'intermédiaire d'un transfert de l'horaire alloué aux activités de français au profit de l'anglais que s'opère cet effet négatif de l'expérimentation sur les acquisitions des élèves en français.

\section{Graphique $\mathbf{n}^{\circ} 2$}

\section{Relation entre durée totale de l'expérimentation EPLV et niveau des élèves en français}

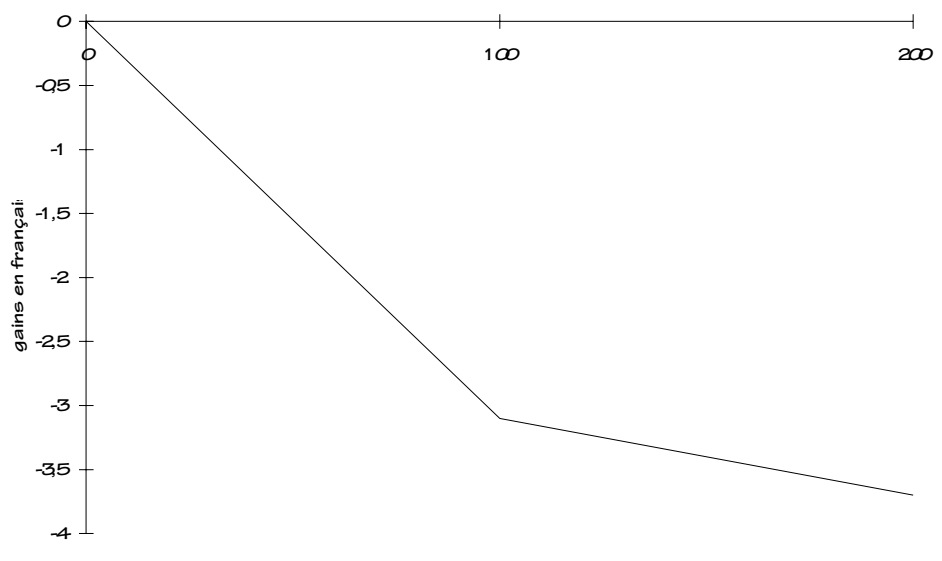

durée de l'initiation en anglais(en heures)

\footnotetext{
${ }^{4}$ Durant la période d'expérimentation certains collèges ont dû "gérer" l'accueil en sixième de ces deux types d'élèves bien que les textes officiels recommandaient d'éviter ces situations.
}

\section{Conclusions}

L'ensemble de ces résultats contribue largement à "mettre à mal" l'opinion selon laquelle il suffirait d'avancer le début de l'apprentissage des langues étrangères, au niveau de l'école primaire, pour que cela produise automatiquement de meilleures acquisitions chez les élèves. Il reste cependant que ces conclusions ne doivent être entendues que dans le cadre strict des "produits" de l'EPLV effectivement évalués par la présente recherche. Notons, par exemple, que les épreuves administrées aux élèves n'ont pas pu évaluer le niveau de production orale de ceux-ci en anglais, ainsi que les acquis de type comportemental que l'initiation a sans doute permis de développer chez eux.

Enfin, il est utile de préciser que, ce dispositif étant nouveau, si la présente recherche a tenté d'évaluer un concept (l'enseignement des langues vivantes à l'école primaire), elle a également évalué, de fait, des pratiques encore mal rodées. De ce point de vue, le fait que l'on ait pu mettre en évidence l'importance des "effets maîtres/classes" sur les acquisitions des élèves constitue plutôt un encouragement à rechercher ce qui, dans les pratiques enseignantes, peut constituer des facteurs propres à améliorer les performances d'un tel dispositif.

Duru-Bellat M. \& Mingat A. (1988), "Le déroulement de la scolarité au collège : le contexte "fait des différences"", Revue Française de Sociologie, Vol. 29, n, pp. 649-666.

O'Neill C. (1993), Les enfants et l'enseignement des langues étrangères - Paris : Credif Hatier/Didier - Coll. "Langues et apprentissage des langues".

Siguan M. \& Mackey W. F. (1986), Education et bilinguisme Paris, Lausanne : UNESCO, Delachaux \& Niestlé.

Pour des résultats plus détaillés...

Genelot S. (1995), L'enseignement des langues à l'école élémentaire : quels acquis pour quels effets au collège

? Eléments d'évaluation: le cas de l'anglais

Cahier de l'Irédu n 58 - Décembre 1995 - 200 pages -

120 Francs (port compris) 


\section{Rappel des dernières Notes}

96/1 Les groupements d'élèves dans l'école primaire rurale en France : efficacité pédagogique et intégration des élèves aux collèges

96/2 Croissance et formation : synthèse critique et test

96/3 Les nouveaux problèmes de l'Ecole en Russie, à la veille du XXIème siècle 